\title{
Advances in polarization sensitive multiphoton nano-bio-imaging
}

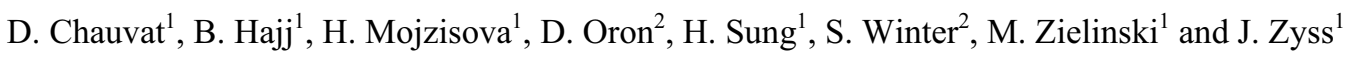 \\ ${ }^{1}$ LPQM (CNRS, UMR 8537) Institut d'Alembert, Ecole Normale Supérieure de Cachan, Cachan, 94230, France \\ ${ }^{2}$ Departement of Physics of Complex Systems, Weizmann Institute of Sciences, Rehovot, Israel
}

\begin{abstract}
In this talk, we shall shortly review four main directions of ongoing research in our laboratories, directed at the conception and demonstration of a variety of innovative configurations in nanoscale multiphoton imaging. A common feature to all of these directions appears to be the central role played by the involvement of polarization features, both in- and outgoing, moreover so in view of the tensorial aspects inherent to nonlinear schemes such second-harmonic generation, electro-optic modulation or two-photon fluorescence which will ne emphasized. These advances relate to the new domain of nonlinear ellipsometry in multiphoton imaging [1], of high relevance to fundamental aspects of nanophotonics and nanomaterial engineering as well as towards basic life science issues. The four domains to be shortly reported are: a) polarization resolved second-harmonic generation in semiconductor QD's with record small sizes in the 10-12 $\mathrm{nm}$ range [2] b) original use of two-photon confocal polarization resolved microscopy in DNA stained by two photon fluorescent dyes in different LC phases arrangements so as to characterize these as well as ascertain the respective DNA-dye orientation (intercalant or groves) [3] c) elaboration and demonstration of an electrooptic confocal microscope in a highly sensitive interferometric and homodyne detection configuration allowing to map weak electric potentials such as in artificial functionalized membranes, the dynamical investigation of firing and propagation aspects of action potentials in neurones being currently the next step [4] d) original plasmon based enhanced nanoscale confocal imaging involving a dual detection scheme (fluorescence imaging and ATR plasmon coupling in reflection) whereby adequate preparation and switching of the incoming polarization state between radial, linear and azimuthal configurations, entail different images and plasmon enhancement levels [5].
\end{abstract}

\section{References}

1. S. Brasselet and J. Zyss, C. R. Physique, 8, 165 (2007)

2. Marcin Zielinski, Small, 191 (2009)

3. H. Mojzisova, J. Olesiak, M. Zielinski, K. Matczyszyn, D. Chauvat, and J. Zyss, Biophys. Journal, 97, 2348 (2009)

4. B. Hajj, S. De Reguardati, L. Hugonin, T. Osaki, S. Takeuchi, B. Le Pioufle, D. Chauvat and J. Zyss, Biophys. Journal, 97, 2913 (2009)

This is an Open Access article distributed under the terms of the Creative Commons Attribution-Noncommercial License 3.0, which permits unrestricted use, distribution, and reproduction in any noncommercial medium, provided the original work is properly cited. 